\title{
In Memoriam Prof. Dr. med. Jürgen Weiss
}

\author{
In Memoriam Prof. Dr. med. Jürgen Weiss
}

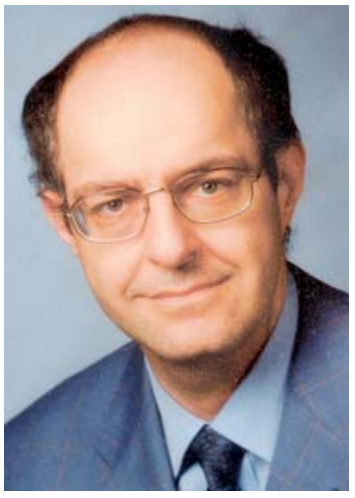

Prof. Dr. med. Jürgen Weiss
Im Alter von 54 Jahren ist auf dem Weltkongress der Deutschen Dermatologen in Seoul Herr Prof. Dr. med. Jürgen Weiss unerwartet verstorben $\left({ }^{*} 23\right.$. Juni $1957, \dagger 23$. Mai 2011).

Seine Jugendzeit verbrachte er in Eberbach am Neckar und begann das Medizinstudium an der Universität Heidelberg - klinischer Teil an der Fakultät für Klinische Medizin Mannheim - mit Abschluss Staatsexamen und Promotion 1982. Es folgte die chirurgische Ausbildung in Bad Dürkheim und eine Forschungszeit in der Immunpharmakologie Hannover. Danach schlug er den Weg in die Dermatologie ein und begann die Facharztausbildung an der Hautklinik Mannheim. Er erwarb die Zusatzbezeichnung Allergologie und wurde 1990 Oberarzt an der Hautklinik Mannheim. Ein Forschungsstipendium führte ihn an das „Ludwig Insititute for Cancer Research“ zur Melanomforschung zunächst nach Montreal, Kanada, und dann nach San Diego, Kalifornien, mit Herrn Prof. W.K. Cavenee. 1993 kehrte er als Oberarzt zurück an die Hautklinik Mannheim und habilitierte 1994 zum Thema Melanom. Im selben Jahr folgte er als Leitender Oberarzt Herrn Prof. A. Kapp an den Lehrstuhl der Medizinischen Hochschule Hannover und erwarb dort 1995 nach Umhabilitation die apl. Professur. Er baute in Hannover neben seinen vielfältigen klinischen Aufgaben die medikamentöse Tumortherapie des Melanoms und ein dermatoonkologisches Forschungslabor auf. Es flossen Fördermittel der DFG und es folgten nationale und internationale Studien. Dennoch entschied er sich
2000 für den Weg in die Niederlassung und einen Praxissitz in Hannover, zusammen mit Herrn Prof. Dietrich Lubach und dann mit Herrn Dr. Kaspari als Praxispartner ab 2006. Er hat die Aufgabe des Fortbildungsreferenten des Landesverbandes Niedersachsens übernommen, war Mitglied des CMEBeirats und Sonderreferent für dermatologische Rezepturen und berufspolitisch hoch engagiert.

Seine wissenschaftlichen Arbeiten umfassen vor allem das Thema Melanom in der Spannbreite der p53-Mutation bis hin zu epidemiologischen Studien zu Risikofaktoren des Melanoms. Dazu kommt eine große Zahl an Arbeiten mit detaillierten Kasuistiken aus der gesamten Spannbreite der Dermatologie und didaktisch hervorragend dargestellte Arbeiten zu STDs - nur als Beispiele.

Als Oberarzt kannte ich ihn in seiner Zeit an der Hautklinik Mannheim bei Herrn Prof. Jung. Wenn ich beschreiben würde, dass er gerne unterrichtete, würde ich ihm nicht gerecht. Was ihn für seine Schüler auszeichnete war ein überdurchschnittliches „didaktisches Eros“ - er liebte es zu lehren, er war begeisternd und mitreißend. Seine unkonventionelle und pragmatische Art hat mich viel gelehrt. Sein Fachwissen war ungewöhnlich breit und fundiert. Sein Engagement für Patienten zeugte von seinem großen Herzen. Seine Integrität empfahl ihn als Kollegen. Er war ein VollblutDermatologe. Bei den Dermatologen, die ihn kannten, wird viel von ihm bleiben - das was er vorgelebt und das was er gelehrt hat.

Christiane Bayerl, Wiesbaden
Bibliografie

DoI http://dx.doi.org/

10.1055/s-0030-1256659

Akt Dermatol 2011; 37:

270 (c) Georg Thieme Verlag KG

Stuttgart · New York

ISSN 0340-2541

Korrespondenzadresse

Prof. Dr. med. Christiane Bayerl

Klinik für Dermatologie

und Allergologie

HSK, Wilhelm-Fresenius-Klinik

Städtisches Klinikum Wiesbaden

Aukammallee 39

65191 Wiesbaden

christiane.bayerl@

hsk-wiesbaden.de 